\title{
28 Research Square \\ The potential role of hsa_circ_0005505 in the rupture of human intracranial aneurysm
}

\section{Xin Chen}

Beijing Tiantan Hospital

Shuzhe Yang

Beijing Tiantan Hospital

Junhua Yang

Beijing Tiantan Hospital

Qingyuan Liu

Beijing Tiantan Hospital

Maogui Li

Beijing Tiantan Hospital

Jun Wu

Beijing Tiantan Hospital

Hao Wang

Beijing Tiantan Hospital

Shuo Wang ( $\nabla$ captain9858@126.com )

Beijing Tiantan Hospital

\section{Research}

Keywords: circular RNA, intracranial aneurysm, vascular smooth muscle cells, phenotype modulation

Posted Date: February 23rd, 2021

DOI: https://doi.org/10.21203/rs.3.rs-210322/v1

License: (a) (i) This work is licensed under a Creative Commons Attribution 4.0 International License. Read Full License 


\section{Abstract \\ Background}

Recently, abundant numbers of studies have revealed many functions of circular RNAs in multiple diseases, however, the role of circular RNA in the rupture of human intracranial aneurysm is still unknown. This study aims to explore the potential functions of circular RNA in the rupture of human intracranial aneurysms.

\section{Results}

The differentially expressed circular RNAs between un-ruptured intracranial aneurysms $(n=5)$ and ruptured intracranial aneurysms $(n=5)$ were analyzed with the Arraystar human circRNAs microarray and total of 13175 circular RNAs were detected. Among them 63 circular RNAs upregulated and 54 circular RNAs downregulated significantly in ruptured intracranial aneurysms compared with un-ruptured intracranial aneurysms $(p<0.05$, Fold Change > 1.5). 5 upregulated circular RNAs were selected for further study (hsa_circ_0001947, hsa_circ_0043001, hsa_circ_0064557, hsa_circ_0058514, hsa_circ_0005505). The results of qPCR showed only hsa_circ_0005505 significantly upregulated $(\mathrm{P}<0.05)$. The expression of hsa_circ_0005505 was higher in ruptured intracranial aneurysm tissues. And our in vitro data showed that hsa_circRNA_005505 promotes the proliferation the migration and apoptosis of vascular smooth muscle cell.

\section{Conclusions}

This study revealed an important role of hsa_circ_0005505 in the proliferation, migration and apoptosis of vascular smooth muscle cell, and indicated that hsa_circ_0005505 may associated with the pathological process of intracranial aneurysms.

\section{Background}

Intracranial aneurysm $(\mathrm{IA})$ is a cerebrovascular disorder characterized by a regional ballooning of intracranial arteries. The incidence of intracranial aneurysm in general population is $1.8 \%-8.4 \%$. Rupture of an intracranial aneurysm can lead to fatal subarachnoid hemorrhage (SAH) which carries a high risk of death or disability. How to assess the possibility of intracranial aneurysm rupture is still inconclusive. In recent 5 years, plenty of researchers studied about the molecular mechanism of the intracranial aneurysm rupture. Many biological processes such as inflammation, cell apoptosis, the phenotypic changes of vascular smooth muscle cell (VSMC), cell adhesion, atherosclerosis and abnormal extracellular matrix metabolism may participate in the mechanism of intracranial aneurysm rupture ${ }^{2,3}$. Existing evidence suggests that the process of abnormal inflammatory response in blood vessel wall is initiated by hemodynamic changes, induces the activation of signaling pathways such as NFKB through unknown mechanism. Then, Matrix Metalloproteinases (MMP) such as MMP2 and MMP9 generates the extracellular matrix degradation and phenotypic changes of VSMC which leads to cell apoptosis ${ }^{4-}$

6 . VSMC distributes in the middle layer of artery wall and is the main cell that synthesizes matrix of artery wall. Loss of components in the middle layer of blood vessels can easily cause the formation and rupture of 
intracranial aneurysms ${ }^{7}$. In the pathological examination of a ruptured intracranial aneurysm, the loss of VSMCs and the thinning of the middle layer of the artery can be seen ${ }^{8}$.

Circle RNAs (termed circRNAs) are one type of non-coding RNAs (ncRNAs) that are formed covalently closed loop structures and widely expressed in human cells ${ }^{9,10}$. In recent years, as the widespread use of RNA sequencing technique and biological experiments, many circRNAs have been discovered and proved to be highly expressed in a tissue-specific or cell type-specific manner ${ }^{11,12}$. And some researchers have revealed the biological function of circRNA. It was reported that circRNAs can function as miRNA sponges that control gene transcription ${ }^{13}$, besides, circRNAs can interact with different proteins and affect the function of combined protein ${ }^{14}$. Moreover, circRNAs have the function of encoding functional peptides or proteins ${ }^{15}, 16$. Emerging evidence also suggests a potential role of circRNAs in different human diseases ${ }^{17}$. However, research about the role of circRNAs in the formation and rupture of intracranial aneurysm are rare, and the overall pathophysiological contributions of circRNAs to intracranial aneurysm remain largely unknown.

In this study we acquired differentially expressed circRNAs between ruptured intracranial aneurysm (RIA)tissues and un-ruptured intracranial aneurysm (UIA) tissues through circRNA microarray. We further detected hsa_circ_0005505 and assessed the role of hsa_circ_0005505 in intracranial aneurysm rupture in vitro.

\section{Results}

\section{Identification of circRNA microarray in human UIA and RIA samples.}

We detected a total of 13174 circRNAs by Arraystar human circRNA Microarray. Among them, 117 circRNAs dysregulated between UIA and RIA tissues(fold-change>1.5, p<0.05) (Additional file 4) and 93 of them have been identified by other studies before. Comparing RIA with UIA tissues, 63 circRNAs upregulated while 54 circRNAs downregulated. All circRNAs we detected contain 5 types: "exonic", "intronic", "antisense", "sense overlapping" and "intergenic". In the 63 upregulated circRNAs, 57(90.4\%) circRNAs arising from the exons of the linear transcript, $4(6.3 \%)$ circRNAs arising from the introns of the linear transcript, and 2(3.3\%) circRNAs transcribed from same gene locus as the linear transcript, but not classified into "exonic" and "intronic" which were classified into "sense overlapping" (Figure 1A). However, in 54 downregulated circRNAs, 45(83.3\%) circRNAs were arising from exons, 4(7.4\%) circRNAs were classified into "sense overlapping", 2(3.7\%) circRNAs were "intronic", 2(3.7\%) circRNAs whose gene locus overlap with the linear RNA but transcribed from the opposite strand which means the "antisense", and 1(1.8\%) circRNA was "intergenic" which means located outside known gene locus (Figure 1B). The variation of circRNA expression between the UIA and RIA samples were assessed (Figure 1C-E). 5 upregulated circRNAs were selected for further investigation (hsa_circ_0001947, hsa_circ_0043001, hsa_circ_0064557, hsa_circ_0058514, hsa_circ_0005505). Besides fold-change $>1.5$ and $p<0.05$, all these 5 circRNAs' raw intensities in UIA and RIA groups were more than 200 and their parental genes were well investigated by other studies in order to reveal these circRNA's functions better.

The circRNA microarray profiling expression results were verified through quantitative reverse transcription PCR (qRT-qPCR) in 5 paired UIA and RIA samples. All these 5 selected circRNAs were upregulated which in agreement with the microarray results, but only hsa_circ_0005505 and hsa_circ_0043001 upregulated significantly $(p<0.05)$ (Figure 1F). Because of the better investigation of their parental genes, hsa_circ_0005505 was finally selected for further research. 


\section{Characteristics and functions of hsa_circ_0005505}

The hsa_circ_0005505(chr12:66597490-66622150) is partly derived from exon 5-11 in human endogenous interleukin 1 receptor associated kinase 3(IRAK3). The genomic sequence of hsa_circ_0005505 is 24660nt and the spliced length is 754nt. To explore the potential functions of hsa_circ_0005505, we found hsa_circ_0005505 contain 14 open reading frames (ORFs) through ORFfinder (https://www.ncbi.nlm.nih.gov/orffinde)(Table 1), but only the longest one has the ability to encode protein. The result of the Conserved Domains (https://www.ncbi.nlm.nih.gov/Structure/cdd/wrpsb.cgi) showed that, the ORF of hsa_circ_0005505 may encode PKC (Protein Kinases, catalytic domain) _like super family (Bit Score=260.20, E-value=4.62e-88). Besides encoding proteins, the other potential function of circRNA is microRNA(miRNA) sponges. Through CSCD, miRNAs binding on hsa_circ_0005505 have been found (Additional file 5). Also, we recognized proteins binding on hsa_circ_0005505 from CSCD, and the result was listed in Table2. Furthermore, fluorescence in situ hybridization (FISH) against hsa_circ_0005505 showed the predominant cytoplasmic distribution of hsa_circ_0005505 (Figure 2A). We also investigated the expression level of hsa_circ_0005505 in HBVSMCs. According to the result of qRTPCR, hsa_circ_0005505 is significantly upregulated in HBVSMCs (Figure 2B).

\section{Construction of circRNA-miRNA-mRNA network and GO, KEGG pathway analysis}

Based on the hsa_circ_0005505 predicted targeting miRNAs, the circRNA-miRNA-mRNA network has been built (Figure3A). Then GO and KEGG pathway analysis of genes in hsa_circ_0005505-miRNA-mRNA network (Additional file 6). have been performed in order to analyze the biological function of these genes. In the ontology analysis of molecular function, hsa_circ_0005505 correlated with genes such as MAP3K2, ACVRL1, CCNT2, RCC2, CTNND1, PTEN, PRKAG1, YWHAZ, CYLD, NR4A3, PDCD10, and mainly enriched in protein binding, DNA binding, RNA polymerase II core promoter proximal region sequence-specific DNA binding, zinc ion binding and sequence-specific DNA binding, respectively (Figure3B). In the biological process of gene oncology, hsa_circ_0005505 mainly correlated with ACVRL1, BTG2, CD86, PPP2CA, NF2, PTPN2 and so on and enriched in positive regulation of transcription, nervous system development, transcription, phosphatidylinositol-mediated signaling and regulation of transcription (Figure3C). In the ontology analysis of cellular component, genes such as CCNT2, CTNND1, DCAF8, MSANTD4, NR3C1, RBPJ, YY1, ELK4, PSMD correlated with hsa_circ_0005505 and mainly enriched in nucleus, nucleoplasm, PcG protein complex, cytoplasm, cytosol, respectively (Figure3D). The result of KEGG revealed that genes correlated with hsa_circ_0005505 enriched in Transcriptional misregulation in cancer, TGF-beta signaling pathway, MAPK signaling pathway, Melanoma and Ras signaling pathway (Figure 3E). In brief, results of GO and KEGG pathway analysis suggested that, genes correlated with hsa_circ_0005505 may associated with cell proliferation and apoptosis.

\section{Knockdown of hsa_circ_0005505 inhibits HBVSMCs proliferation, migration and induces apoptosis in virto}

The pictures of infected HBVSMCs were shown in Figure 4A. Then qPCR was performed to evaluate the transduction efficiency of three virus (Figure 4B). Based on the result of qPCR, HBVSMCs infected by LV-circRNARNAi (74402-1) were selected for further experiments. Knockdown of hsa_circ_0005505 markedly inhibits HBVSMCs proliferation after transfected by shRNA according to the MTT assay (Figure 4C) which was in consistent with the result of CCK8 assay (Figure 4D). The effect of hsa_circ_0005505 on apoptosis of HBVSMCs was also analyzed. The result of apoptosis analysis suggested that knockdown of hsa_circ_0005505 significantly promotes HBVSMCs apoptosis (Figure 4E,4F). Besides, the Wound-healing assay demonstrated that hsa_circ_0005505 silencing significantly impeded HBVSMCs migration (Figure 4G). 


\section{Disscussion}

The development of high-throughput sequencing, gene chip technology and bioinformatics over the last decade have largely improved our knowledge on circRNA. It is becoming increasingly clear that circRNAs play a crucial role in the pathological process of many kinds of cancers, such as colorectal cancer ${ }^{21}$, breast cancer ${ }^{22}$, and hepatocellular carcinoma ${ }^{23}$. However, whether circRNA participate in the pathological process of intracranial aneurysm is still unknown. In this study, we found hsa_circ_0005505 significantly upregulated in ruptured intracranial aneurysm tissues. Characteristics of hsa_circ_0005505 have been revealed and bioinformatical analysis of hsa_circ_0005505 also have been performed. Knockdown of hsa_circ_0005505 inhibits the proliferation, migration and induces apoptosis of HBVSMCs, which means hsa_circ_0005505 promotes the proliferation, migration of HBVSMCs but suppresses their apoptosis. According to research about the formation and rupture of intracranial aneurysm, smooth muscle cells and phenotypic modulation play a significant role in these processes ${ }^{24}$. In the formation of intracranial aneurysm, the VSMCs migrate into the intima and proliferate producing myointimal hyperplasia ${ }^{25}$. Following phenotypic modulation during intracranial aneurysm formation, VSMCs may finally lose their phenotype and undergo apoptosis leading to aneurysm rupture ${ }^{8}$. In the early stage of intracranial aneurysm formation, VSMCs were modulated from differentiated VSMC concerned with contraction characterized by myosin heavy chain (MHC) and vascular smooth muscle a-actin to cells with a proinflammatory, pro-matrix remodeling phenotype characterized by the increased expression of inflammatory factors and MMP ${ }^{26-29}$. Based on these studies, we make a hypothesis that hsa_circ_0005505 may play a pivotal role in the phenotypic modulation of HBVSMCs. The differential expression of markers for phenotypes of VSMCs in HBVSMCs and hsa_circ_0005505 silenced HBVSMCs will be verified in our further study.

Research about the role of circRNAs in the formation and rupture of intracranial aneurysm is still scarce. However, several circRNAs have been found to be related with VSMCs. Holdt et al ${ }^{30}$ demonstrated that circ_ANRIL inhibits the proliferation and promotes the apoptosis of VSMCs and is associated with coronary artery disease. Hall et al ${ }^{31}$ found circ_Lrp6 is a sponge for miR-145 and circ_Lrp6 hindered miR-145-mediated regulation of VSMC migration, proliferation, and differentiation, furthermore, the ratio of circ_Lrp6 bound to miR-145 versus unbound could play a role in vascular pathogenesis. CircRNAs mainly regulate gene transcription by acting as miRNA sponges, miRNAs binding with hsa_circ_0005505 have been revealed and we also have constructed the hsa_circ_0005505-miRNA-mRNA network. We will further explore the interaction of hsa_circ_0005505 with miRNAs and reveal the precise function of hsa_circ_0005505 in the pathological process of intracranial aneurysm.

Besides function as miRNA sponges to control gene transcription, circRNAs' ability of encoding peptides or proteins also have been studied ${ }^{15,16}$. We have found that the ORF of hsa_circ_0005505 may encode PKC_like super family through bioinformatic analysis. PKC_like super family contains at least 11 isozymes and can be classified into 3 groups $^{32}$. Existing studies demonstrate that PKC promotes the proliferation, migration and dedifferentiation of VSMCs ${ }^{33-36}$ and this is in line with our results about the function of hsa_circ_0005505 in virto. During the pathological process of intracranial aneurysm, the VSMC can undergo phenotype modulation. With the stimulation of environment, VSMC can transform from a differentiated phenotype concerned mainly with contraction to an undifferentiated, pro-inflammatory, promatrix -remodeling phenotype ${ }^{24}$. Furthermore, Zuniga et $\mathrm{al}^{37}$ found that a member of PKC_like super family, PKC-epsilon, is a key mediator in resistin-induced 
inflammation by promoting the activation of NF-KB. All these findings suggest that PKC may play an important role in the formation and rupture of intracranial aneurysm and we'll verify this hypothesis in our further study.

Our study also has some limitations. Firstly, the amount of our tissue sample is small. Our results need more intracranial aneurysm samples to testify. Second, due to the rare of aneurysm samples, some of our samples were stored in liquid nitrogen for several weeks, perhaps this would affect the amount of circRNAs. Lastly, the lack of studies about circRNAs in aneurysm make us can't compare our results with others in order to improve our methods.

\section{Conclusions}

The above findings revealed an important role of hsa_circ_0005505 in the proliferation, migration and apoptosis of VSMCs and may take part in the phenotype modulation of VSMCs, indicating that hsa_circ_0005505 may associated with the rupture of intracranial aneurysms.

\section{Methods}

\section{Patients and specimens}

A total of 10 pairs of ruptured and un-ruptured intracranial aneurysm tissues were obtained from surgical resection during the aneurysm clipping surgery in Beijing Tiantan Hospital. The collection of human specimens was approved by the Medical Ethics Committee of Beijing Tiantan Hospital, Capital Medical University. Written informed consent was obtained from each patient according to the policies of the committee. All specimens were stored in liquid nitrogen, and 5 pairs of samples were used to conduct circRNA microarray analysis, other samples were used to perform qPCR.

\section{Total RNA isolation and quality control}

We extracted RNA with the use of Trizol Reagent (Invitrogrn, NY, USA) from 5 paired ruptured and un-ruptured intracranial aneurysms according to the manufacturer's instructions. The quality and concentration of RNA was tested by the NanoDrop ND- 1000 (Thermo Fisher Scientific, Wilmington, DE, USA) (Additional file 1).

\section{RNA labeling and hybridization}

Sample labeling and array hybridization were performed according to the manufacturer's protocol (Arraystar Inc.). Briefly, total RNAs were digested with Rnase R (Epicentre, Inc.) to remove linear RNAs and enrich circular RNAs. Then, the enriched circular RNAs were amplified and transcribed into fluorescent cRNA utilizing a random priming method (Arraystar Super RNA Labeling Kit; Arraystar). The labeled cRNAs (Additional file 2) were purified by RNeasy Mini Kit (Qiagen). The concentration and specific activity of the labeled cRNAs (pmol Cy3/ $\mu$ g cRNA) were measured by NanoDrop ND-1000. $1 \mu \mathrm{g}$ of each labeled cRNA was fragmented by adding $5 \mu 10 \times$ Blocking Agent and $1 \mu \mathrm{l}$ of $25 \times$ Fragmentation Buffer, then heated the mixture at $60{ }^{\circ} \mathrm{C}$ for 30 min, finally $25 \mu \mathrm{l} \times$ Hybridization buffer was added to dilute the labeled cRNA. $50 \mu$ of hybridization solution was dispensed into the gasket slide and assembled to the circRNA expression microarray slide. The slides were incubated for 17 hours at $65^{\circ} \mathrm{C}$ in an Agilent Hybridization Oven. The hybridized arrays were washed, fixed and scanned using the Agilent Scanner G2505C. 


\section{CircRNA microarray analysis}

Agilent Feature Extraction software (version 11.0.1.1) was used to analyze acquired array images. Quantile normalization and subsequent data processing were performed using the $\mathrm{R}$ software limma package. Differentially expressed circRNAs with statistical significance between two groups were identified through Volcano Plot filtering. Differentially expressed circRNAs between two samples were identified through Fold Change filtering. Hierarchical Clustering was performed to show the distinguishable circRNAs expression pattern among samples.

\section{Real-time quantitative PCR (qPCR)}

Real-time PCR was used to verify differentially expressed circRNAs obtained from microarray analysis. RNase R ( Lucigen, $20 \mathrm{U}, 37^{\circ} \mathrm{C} \varangle 3 \mathrm{~h}$ ) was used to purify the circRNAs again. The relevant cDNAs were composed (M-MLV, promega ) and stored in $-20^{\circ} \mathrm{C}$. QuantStudio5 Real-time PCR System (Applied Biosystems) was used to perform qPCR. The sequence of circRNA results was acquired from the database "circBase" (http://circrna.org). Primers were produced by RiboBio (Guangzhou, China) (Additional file3). Because of the influence of concentration quantitative error and reverse transcription efficiency error, the cDNA content of every sample was different. In order to correct these errors, we regarded housekeeping gene $\beta$-actin as internal reference, as a result, we accepted the ratio of genes to be tested and internal reference, in other words, the relative content of gene to be tested.

\section{Construction of circRNA-miRNA-mRNA network}

CSCD (http://gb.whu.edu.cn/CSCD/) was used to recognize miRNAs binding on our target circRNA. Three algorithms including Targetscan ${ }^{18}$, miRDB $^{19}$, and miRTarBase ${ }^{20}$ were used to analyze parental genes of miRNAs binding on the target circRNA. The circRNA-miRNA-mRNA network was visualized by Cytoscape(version3.7.1).

\section{Gene ontology (GO) and Kyoto Encyclopedia of Genes and Genomes (KEGG) pathway analysis}

We assumed that our target circRNA may have molecular interactions with these genes, or our target circRNA may regulate biological functions through these genes. GO analysis on genes correlated with these miRNAs was performed by DAVID (https://david.ncifcrf.gov/). The P value after adjustment represents the significance of GO terms. We also perform KEGG pathway analysis of parental genes of circRNA-binding miRNAs, in order to reveal the biological or pathological processes which circRNAs participate in. The $P$ value after adjustment represents the significance of pathway correlations as well.

\section{Cell culture}

Human brain vascular smooth muscle cells (HBVSMCs) were obtained from Bnbio (BNCC102172, Beijing, China). The cells were cultured in Smooth Muscle Cell Medium (SMCM) containing 2\% fetal bovine serum (FBS), $5 \mathrm{ml}$ of smooth muscle cell growth supplement (SMCGS, Cat. No. 1152), and $5 \mathrm{ml}$ of penicillin/streptomycin solution (P/S, Cat. No. 0503$)$ at $37^{\circ} \mathrm{C}$ in an incubator of $5 \% \mathrm{CO} 2$.

\section{Transduction of cells}


The hsa_circ_0005505 specific shRNA, their relevant lentiviruses (LV-circRNA-RNAi (74402-1), LV-circRNA-RNAi (74403-2), LV-circRNA-RNAi (74404-1)) and negative control lentivirus were obtained from Shanghai Genechem Co, LTD (Shanghai, China). HBVSMCs were transduced with individual types of lentivirus at a multiplicity of infection (MOI) of 50 and the ideal value of infection efficiency was $80 \%$.

\section{MTT assay}

MTT assay was used to measure the proliferation of HBVSMCs according to the manufacturer's instructions (Dingguo Biotech, Shanghai, China). HBVSMCs $\left(2 \times 10^{3}\right.$ per well) were plate in 96 -well plates and treated with $20 \mu \mathrm{l}$ of $5 \mathrm{mg} / \mathrm{ml}$ MTT solution, then the spectrophotometrically at $490 \mathrm{~nm}$ and $570 \mathrm{~nm}$ was analyzed by automatic microplate reader (Tecan infinite).

\section{Cell counting kit-8 (CCK-8) assay}

CCK-8 assay was used to test the cell viability in order to verify the effect of the target gene on cell proliferation. The assay was performed according to the manufacturer's instructions (Dojindo Laboratories, Kumamoto, Japan). HBVSMCs $\left(2 \times 10^{3}\right.$ per well) were plate in 96 -well plates and treated with $10 \mu$ l of CCK-8 solution, then the spectrophotometrically at $450 \mathrm{~nm}$ was analyzed by automatic microplate reader (Tecan infinite).

\section{Apoptosis analysis}

Cell apoptosis was analyzed in two ways. The caspase3/7 assay was used to verify the effect of the target gene on apoptosis of cells by using Caspase-Glo 3/7 Assay reagent test kit (Promega). We also analyzed cell apoptosis by using the Annexin V-APC Apoptosis Detection Kit (eBiosciences \#88-8007) according to the manufacturer's instruction. HBVSMCs were stained with APC and then analyzed by fluorescence-activated cell sorting using FACScan (BD Biosciences).

\section{Wound- healing assay}

The wound -healing assay was used to measure the migration rate of cells. The transfected HBVSMCs were seeded into 96 well plates $\left(5 \times 10^{4}\right.$ per well). After $24 \mathrm{~h}$ incubation, parallel wounds with similar width were made in each well by 96 Wounding Replicator (VP scientific). Wound closure level was monitored by Celigo (Nexcelom) in $0 \mathrm{~h}, 8 \mathrm{~h}, 24 \mathrm{~h}$ after wounded and lastly analyze the migration rate.

\section{Statistical analysis}

The fold-changes were estimated by unpaired Student's t-test and used to identify the differentially expressed circRNAs in the sample of intracranial aneurysms. CircRNAs were selected as differentially expressed with a $P<0.05$ and a fold-change $>1.5$, which means they were statistically significant. The significance of qPCR was assessed by Student's t-test and $\mathrm{P}<0.05$ was considered statistically significant, it was analyzed by GraphPad Prism 8.4.0 (GraphPad Software, La Jolla, CA, USA). Other statistical methods such as chi-squared test, Wilcoxon signed-rank test and Mann Whitney U test were also performed. All statistical analyses were performed by SPSS 19.0 (SPSS, Inc., Chicago, IL).

\section{Abbreviations}


circRNA

circular RNA

$\mathrm{SAH}$

subarachnoid hemorrhage

VSMC

vascular smooth muscle cell

HBVSMC

human brain vascular smooth muscle cell

RIA

ruptured intracranial aneurysm

UIA

unruptured intracranial aneurysm

\section{Declarations}

Ethics approval and consent to participate:

This study was approved by the institutional review board of the Beijing Tiantan hospital (Ethical No. KY2017076-01). Privacy of patients was efffectively protected.

\section{Consent for publication!}

Not applicable

\section{Availability of data and materials $\square$}

The datasets used and/or analysed during the current study are available from the corresponding author on reasonable request.

\section{Competing interests $\square$}

The authors declare that they have no competing interests.

\section{Funding:}

This work was supported by the National Natural Science Foundation of China (Grant No. 81671129, S.W.).

\section{Authors' contributions:}

SW and HW was in charge of supervising the whole study. XC contributed to the conception or design of the work. XC and SY were responsible for drafting and revising. QL, ML, JW and JY were responsible for analysis and interpretation of data. All authors contributed to manuscript revision, read, and approved the submission.

\section{Acknowledgements:}

Not applicable 


\section{References}

1. Kang HG, Kim BJ, Lee J, Kim MJ, Kang DW, Kim JS, et al. Risk factors associated with the presence of unruptured intracranial aneurysms. Stroke. 2015;46:3093-8.

2. Jabbarli R, Rauschenbach L, Dinger TF, Darkwah Oppong M, Rodemerk J, Pierscianek D, et al. In the wall lies the truth: A systematic review of diagnostic markers in intracranial aneurysms. Brain Pathol. 2020;30:43745.

3. Jiang P, Wu J, Chen X, Ning B, Liu Q, Li Z, et al. Quantitative proteomics analysis of differentially expressed proteins in ruptured and unruptured cerebral aneurysms by itraq. J Proteomics. 2018;182:45-52.

4. Cheng WT, Wang N. Correlation between mmp-2 and nf-k b expression of intracranial aneurysm. Asian Pac J Trop Med. 2013;6:570-3.

5. Lai XL, Deng ZF, Zhu XG, Chen ZH. Apc gene suppresses intracranial aneurysm formation and rupture through inhibiting the nf-kb signaling pathway mediated inflammatory response. Biosci Rep. 2019;39.

6. Liu Q, Wang Q, Li H. Embelin inhibits abdominal aortic aneurysm through decreasing il6induced stat3 and nfkb inactivation. Mol Med Rep. 2018;18:2365-72.

7. Chalouhi N, Hoh BL, Hasan D. Review of cerebral aneurysm formation, growth, and rupture. Stroke. 2013;44:3613-22.

8. Chalouhi N, Ali MS, Jabbour PM, Tjoumakaris SI, Gonzalez LF, Rosenwasser RH, et al. Biology of intracranial aneurysms: Role of inflammation. J Cereb Blood Flow Metab. 2012;32:1659-76.

9. Li J, Yang J, Zhou P, Le Y, Zhou C, Wang S, et al. Circular rnas in cancer: Novel insights into origins, properties, functions and implications. Am J Cancer Res. 2015;5:472-80.

10. Salzman J, Gawad C, Wang PL, Lacayo N, Brown PO. Circular rnas are the predominant transcript isoform from hundreds of human genes in diverse cell types. PLoS One. 2012;7:e30733.

11. Liang D, Wilusz JE. Short intronic repeat sequences facilitate circular rna production. Genes Dev. 2014;28:2233-47.

12. Starke S, Jost I, Rossbach O, Schneider T, Schreiner S, Hung LH, et al. Exon circularization requires canonical splice signals. Cell Rep. 2015;10:103-11.

13. Hansen TB, Jensen TI, Clausen BH, Bramsen JB, Finsen B, Damgaard CK, et al. Natural rna circles function as efficient microrna sponges. Nature. 2013;495:384-8.

14. Du WW, Zhang C, Yang W, Yong T, Awan FM, Yang BB. Identifying and characterizing circrna-protein interaction. Theranostics. 2017;7:4183-91.

15. Pamudurti NR, Bartok O, Jens M, Ashwal-Fluss R, Stottmeister C, Ruhe L, et al. Translation of circrnas. Mol Cell. 2017;66:9-21.e27.

16. Yang $Y$, Fan X, Mao M, Song X, Wu P, Zhang Y, et al. Extensive translation of circular rnas driven by $n(6)$ methyladenosine. Cell Res. 2017;27:626-41.

17. Chen Y, Li C, Tan C, Liu X. Circular rnas: A new frontier in the study of human diseases. J Med Genet. 2016;53:359-65.

18. Lewis BP, Shih IH, Jones-Rhoades MW, Bartel DP, Burge CB. Prediction of mammalian microrna targets. Cell. 2003;115:787-98. 
19. Wong N, Wang X. Mirdb. An online resource for microrna target prediction and functional annotations. Nucleic Acids Res. 2015;43:D146-52.

20. Hsu SD, Lin FM, Wu WY, Liang C, Huang WC, Chan WL, et al. Mirtarbase: A database curates experimentally validated microrna-target interactions. Nucleic Acids Res. 2011;39:D163-9.

21. Zeng K, Chen X, Xu M, Liu X, Hu X, Xu T, et al. Circhipk3 promotes colorectal cancer growth and metastasis by sponging mir-7. Cell Death Dis. 2018;9:417.

22. Liu Z, Zhou Y, Liang G, Ling Y, Tan W, Tan L, et al. Circular rna hsa_circ_001783 regulates breast cancer progression via sponging mir-200c-3p. Cell Death Dis. 2019;10:55.

23. Yu J, Xu QG, Wang ZG, Yang Y, Zhang L, Ma JZ, et al. Circular rna csmarca5 inhibits growth and metastasis in hepatocellular carcinoma. J Hepatol. 2018;68:1214-27.

24. Starke RM, Chalouhi N, Ding D, Raper DM, McKisic MS, Owens GK, et al. Vascular smooth muscle cells in cerebral aneurysm pathogenesis. Transl Stroke Res. 2014;5:338-46.

25. Kosierkiewicz TA, Factor SM, Dickson DW. Immunocytochemical studies of atherosclerotic lesions of cerebral berry aneurysms. J Neuropathol Exp Neurol. 1994;53:399-406.

26. Aoki T, Kataoka H, Moriwaki T, Nozaki K, Hashimoto N. Role of timp-1 and timp-2 in the progression of cerebral aneurysms. Stroke. 2007;38:2337-45.

27. Aoki T, Kataoka H, Nishimura M, Ishibashi R, Morishita R, Miyamoto S. Ets-1 promotes the progression of cerebral aneurysm by inducing the expression of mcp-1 in vascular smooth muscle cells. Gene Ther. 2010;17:1117-23.

28. Ishibashi M, Egashira K, Zhao Q, Hiasa K, Ohtani K, Ihara Y, et al. Bone marrow-derived monocyte chemoattractant protein-1 receptor ccr2 is critical in angiotensin ii-induced acceleration of atherosclerosis and aneurysm formation in hypercholesterolemic mice. Arterioscler Thromb Vasc Biol. 2004;24:e174-8.

29. Kilic T, Sohrabifar M, Kurtkaya O, Yildirim O, Elmaci I, Günel M, et al. Expression of structural proteins and angiogenic factors in normal arterial and unruptured and ruptured aneurysm walls. Neurosurgery. 2005;57:997-1007. discussion 1997 - 1007.

30. Holdt LM, Stahringer A, Sass K, Pichler G, Kulak NA, Wilfert W, et al. Circular non-coding rna anril modulates ribosomal rna maturation and atherosclerosis in humans. Nat Commun. 2016;7:12429.

31. Hall IF, Climent M, Quintavalle M, Farina FM, Schorn T, Zani S, et al. Circ_Irp6, a circular rna enriched in vascular smooth muscle cells, acts as a sponge regulating mirna-145 function. Circ Res. 2019;124:498-510.

32. Yamamoto M, Acevedo-Duncan M, Chalfant CE, Patel NA, Watson JE, Cooper DR. Acute glucose-induced downregulation of pkc-betaii accelerates cultured vsmc proliferation. Am J Physiol Cell Physiol. 2000;279:C587-95.

33. Rasmussen H, Forder J, Kojima I, Scriabine A. Tpa-induced contraction of isolated rabbit vascular smooth muscle. Biochem Biophys Res Commun. 1984;122:776-84.

34. Sobey CG, Faraci FM. Subarachnoid haemorrhage: What happens to the cerebral arteries? Clin Exp Pharmacol Physiol. 1998;25:867-76.

35. Ding Q, Chai H, Mahmood N, Tsao J, Mochly-Rosen D, Zhou W. Matrix metalloproteinases modulated by protein kinase $c \varepsilon$ mediate resistin-induced migration of human coronary artery smooth muscle cells. J Vasc Surg. 2011;53:1044-51. 
36. Calabro P, Samudio I, Willerson JT, Yeh ET. Resistin promotes smooth muscle cell proliferation through activation of extracellular signal-regulated kinase 1/2 and phosphatidylinositol 3-kinase pathways.

Circulation. 2004;110:3335-40.

37. Zuniga MC, Raghuraman G, Hitchner E, Weyand C, Robinson W, Zhou W. Pkc-epsilon and tlr4 synergistically regulate resistin-mediated inflammation in human macrophages. Atherosclerosis. 2017;259:51-9.

\section{Tables}

Table1. Open reading frames that detected from hsa_circ_0005505

\begin{tabular}{|c|c|c|c|c|c|c|}
\hline Label & Strand & Frame & Start & Stop & Length(nt|aa) & Nucleotide sequence \\
\hline ORF1 & + & 1 & 259 & 345 & $87 \mid 28$ & MSPWIMFLFLNIMKKEYCLNLPSAFKIS \\
\hline ORF 2 & + & 1 & 433 & 543 & $111 \mid 36$ & MLSNYLNRRKKCSVRSIGRGFYLSLKFYYCFITQTY \\
\hline ORF3 & + & 2 & 656 & $>754$ & $99 \mid 32$ & MAHSNRYINRNIQSHSLPAQRSTMLGHLWQYIK \\
\hline \multirow[t]{5}{*}{ ORF4 } & + & 3 & 24 & $>752$ & $729 \mid 242$ & MDVRHIEKYVDQGKSGTRELLWSWAQKNKTIGDLLQVLQEMGHRRAIHLI \\
\hline & & & & & & TNYGAVLSPSEKSYQEGGFPNILFKETANVTVDNVLIPEHNEKGILLKSS \\
\hline & & & & & & ISFQNIIEGTRNFHKDFLIGEGEIFEVYRVEIQNLTYAVKLFKQEKKMQC \\
\hline & & & & & & KKHWKRFLSELEVLLLFHHPNILELAAYFTETEKFCLIYPYMRNGTLFDR \\
\hline & & & & & & LQCVGDTAPLPWHIRIGILIGISKAIHYLHNVQPCSVICGSIS \\
\hline ORF5 & - & 1 & 700 & 587 & $114 \mid 37$ & MALDIPINIPIRMCQGSGAVSPTHCNLSKSVPFLMYG \\
\hline ORF 6 & - & 1 & 409 & 332 & $78 \mid 25$ & MYTSKISPSPIRKSLWKFLVPSMIF \\
\hline ORF7 & - & 1 & 328 & 215 & $114 \mid 37$ & MMEDLSSIPFSLCSGIRTLSTVTLAVSLNNIFGNPPS \\
\hline ORF 8 & - & 1 & 115 & 35 & $81 \mid 26$ & MVLFFCAQDQSNSLVPLLPWSTYFSI \\
\hline ORF9 & - & 2 & 726 & 559 & $168 \mid 55$ & MVERCAGSEWLWIFLLIYRFECAKGVGPCHLHTAICQKVFHFSCMDKSDRTSQSL \\
\hline ORF10 & - & 2 & 555 & 448 & $108 \mid 35$ & MQPTLVCLGDETVVKLQAQIKTSSNASYTAFFSPV \\
\hline ORF11 & - & 2 & 432 & 352 & $81 \mid 26$ & MLGFESPLCIPQKSLLLQLGSLCGNF \\
\hline ORF12 & - & 2 & 75 & $>1$ & $75 \mid 24$ & MFHFYLGLHTFQYDEHPASCLKVSL \\
\hline ORF13 & - & 3 & 629 & 540 & $90 \mid 29$ & MQSVKKCSISHVWINQTELLSLCKICSQL \\
\hline ORF14 & - & 3 & 335 & 240 & $96 \mid 31$ & MKADGRFKQYSFFIMFRNKNIIHGDIGCFLE \\
\hline
\end{tabular}

ORF: open reading frame. 
Table2. Proteins binding on hsa_circ_0005505.

\begin{tabular}{|c|c|c|c|c|}
\hline ID & $\mathrm{RBP}$ & Start & End & Details \\
\hline 1 & FUS_Human_GSE43308_HITS-CLIP & 66605210 & 66605225 & HHMF2_86273_FUS_rep2_1 \\
\hline 2 & FUS_Human_GSE43308_HITS-CLIP & 66604094 & 66604110 & HHMF2_86272_FUS_rep2_2 \\
\hline 3 & FUS_Human_GSE43308_HITS-CLIP & 66621697 & 66621715 & HHMF2_86276_FUS_rep2_4 \\
\hline 4 & DGCR8_Human_GSE39086_HITS-CLIP & 66608982 & 66609004 & HHCT1_6374_DGCR8_T7.1_1 \\
\hline 5 & FUS_Human_GSE43308_HITS-CLIP & 66619959 & 66619978 & HHMF2_86275_FUS_rep2_1 \\
\hline 6 & FUS_Human_GSE43308_HITS-CLIP & 66603282 & 66603300 & HHMF3_96612_FUS_rep3_3 \\
\hline 7 & FUS_Human_GSE43308_HITS-CLIP & 66611178 & 66611193 & HHMF3_96617_FUS_rep3_1 \\
\hline 8 & FUS_Human_GSE43308_HITS-CLIP & 66604013 & 66604032 & HHMF3_96613_FUS_rep3_3 \\
\hline 9 & FUS_Human_GSE43308_HITS-CLIP & 66609043 & 66609058 & HHMF3_96616_FUS_rep3_1 \\
\hline 10 & FUS_Human_GSE43308_HITS-CLIP & 66606215 & 66606247 & HHMF3_96615_FUS_rep3_3 \\
\hline 11 & FUS_Human_GSE43308_HITS-CLIP & 66604755 & 66604771 & HHMF3_96614_FUS_rep3_3 \\
\hline 12 & DGCR8_Human_GSE39086_HITS-CLIP & 66618821 & 66618842 & HHCD1_11057_DGCR8_D8.1_1 \\
\hline
\end{tabular}

RBP: RNA Binding Proteins

\section{Figures}


A

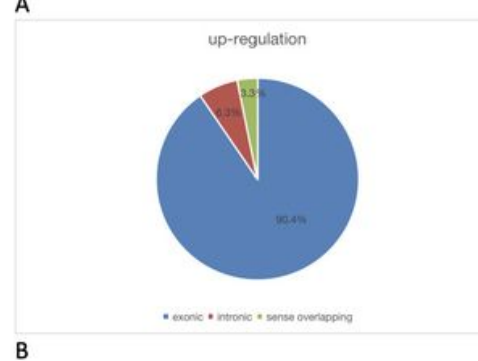

B

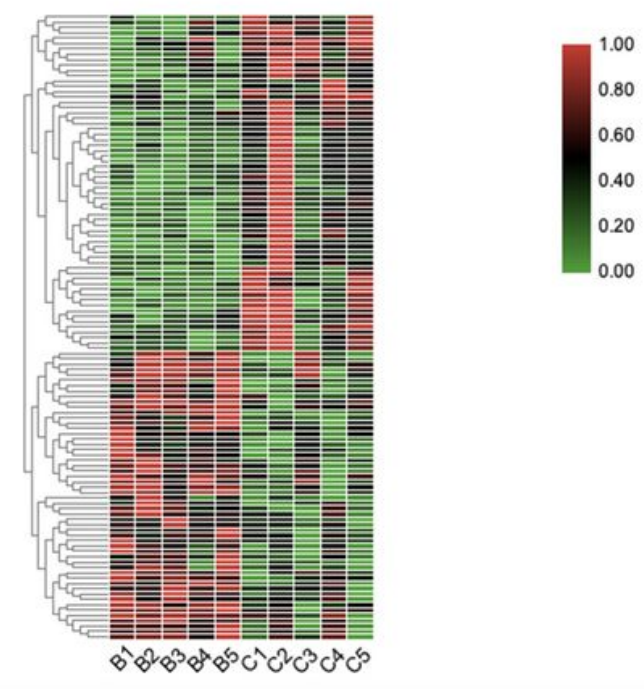

c

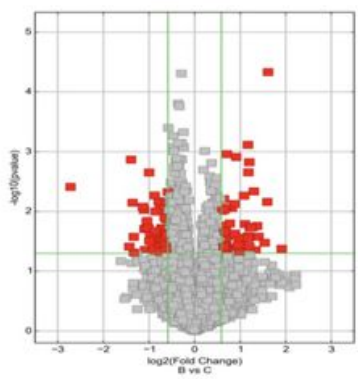

D

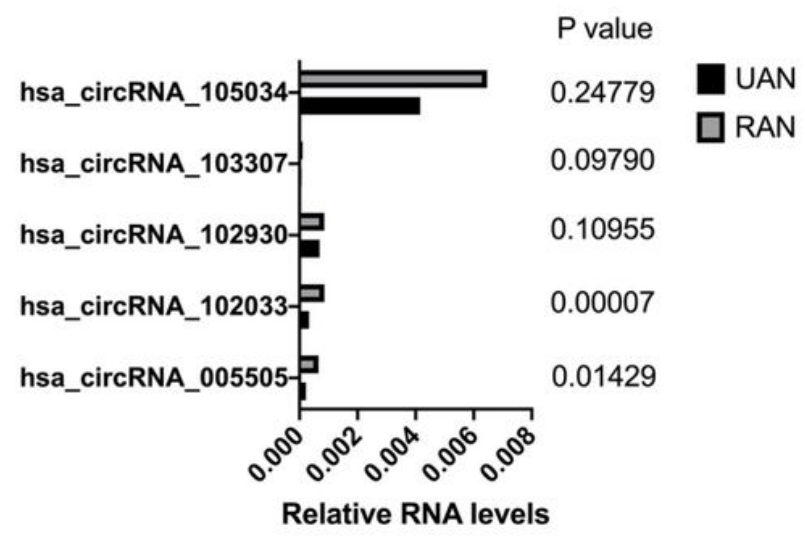

\section{Figure 1}

Differential expression of circRNAs in intracranial aneurysm tissues. A. The constituent of dysregulated circRNAs. B.The hierarchical clustering of differentially expressed circRNAs. 'Red' indicates high relative expression, and 'green' indicates low relative expression. Group B represented unruptured intracranial aneurysm samples, group C represented ruptured intracranial aneurysm samples. C. Volcano Plots are used for visualizing differential expression between two different conditions. The vertical lines correspond to 1.5-fold up and down, respectively, and the horizontal line represents a p-value of 0.05 . So $\$ the red point in the plot represents the differentially expressed circRNAs with statistical significance. Group B represented unruptured intracranial aneurysm samples, group $C$ represented ruptured intracranial aneurysm samples. D. Validation of the differential expression of five upregulated circRNAs. 
Merge

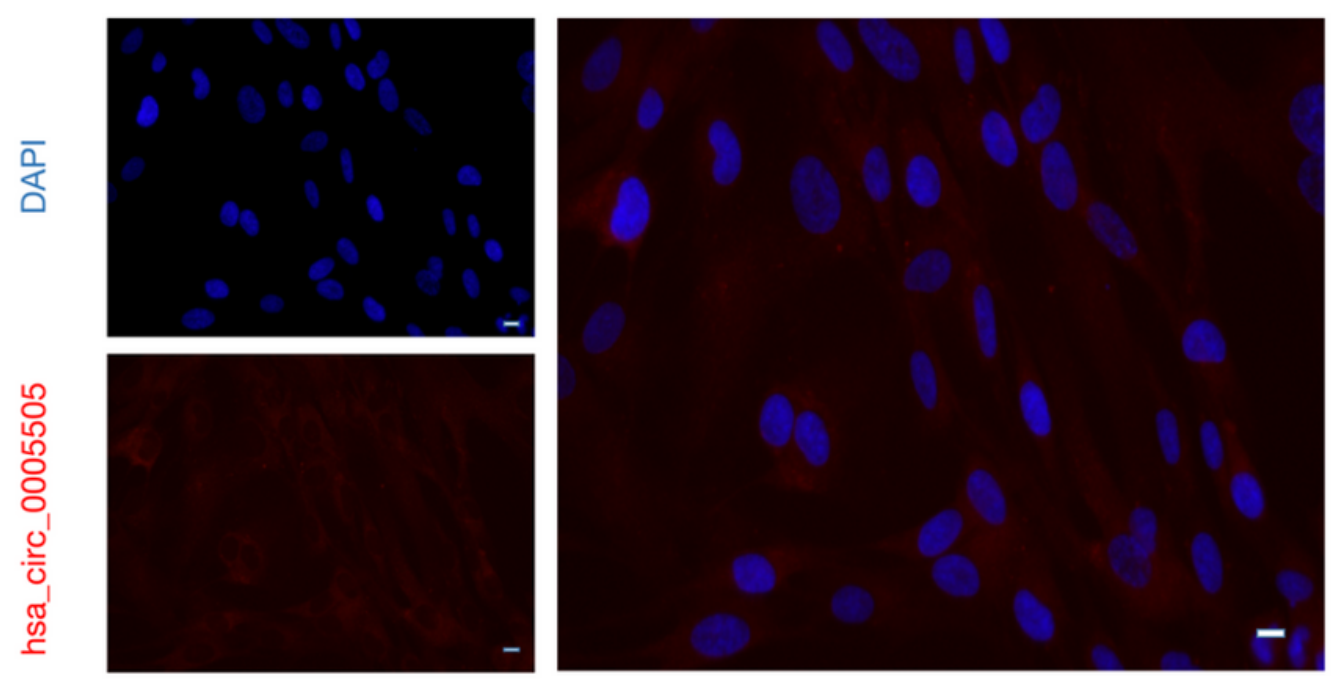

B

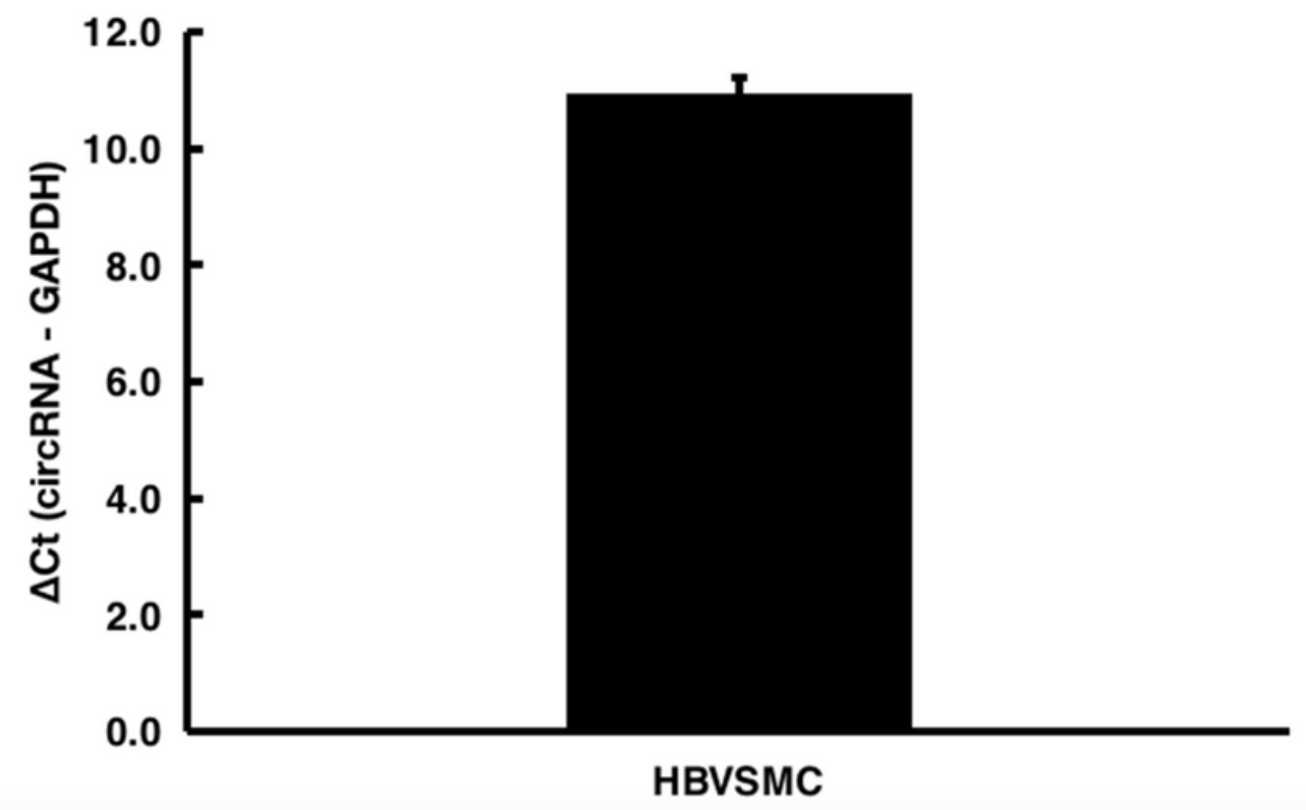

Figure 2

Hsa_circ_0005505 is upregulated in HBVSMCs. A. Fluorescence in situ hybridization assay showing cytoplasmic localization of hsa_circ_0005505 in HBVSMCs. B. Quantitative real-time PCR analysis of hsa_circ_0005505 expression level in HBVSMCs. GAPDH was regarded as the reference gene. $\triangle \mathrm{Ct}$ value $=\mathrm{Ct}$ value of target gene Ct value of reference gene. $\triangle \mathrm{Ct}$ value $\leq 12$ represents the high expression of target gene in cells, $\triangle \mathrm{Ct}$ value $\geq 16$ represents the low expression of target gene in cells. Scale bar $=25 \mu \mathrm{m}$. 

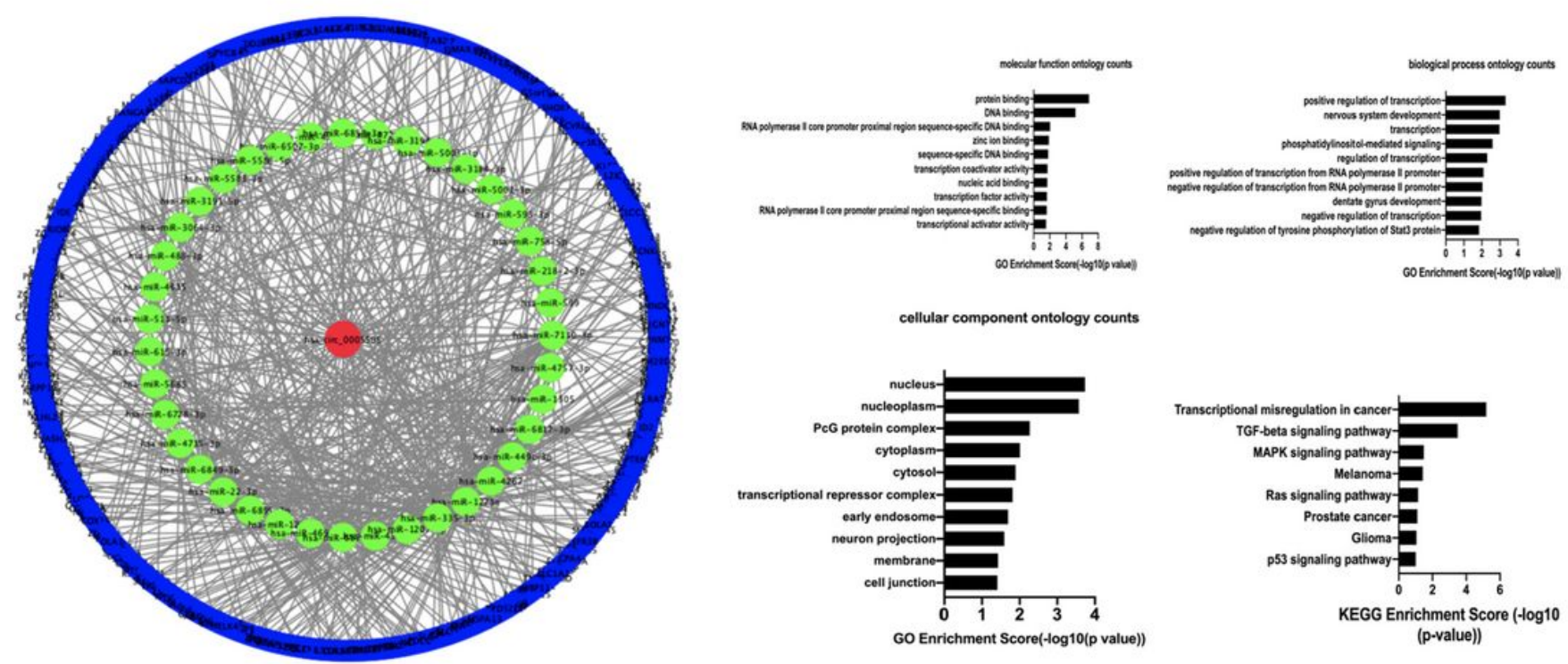

Figure 3

GO and KEGG pathway analysis of hsa_circ_0005505 co-expressed genes. A. Constructed hsa_circ_0005505miRNA-mRNA network. Red ellipse: hsa_circ_0005505, green ellipse: miRNAs potentially interacted with hsa_circ_0005505, blue ellipse: genes positively co-expressed with hsa_circ_0005505. B. The molecular function ontology analysis $\varangle$ biological process ontology analysis $₫$ cellular component ontology analysis and KEGG pathway analysis of hsa_circ_0005505. GO enrichment score and KEGG enrichment score represent the number of genes enriched in the cluster.

A
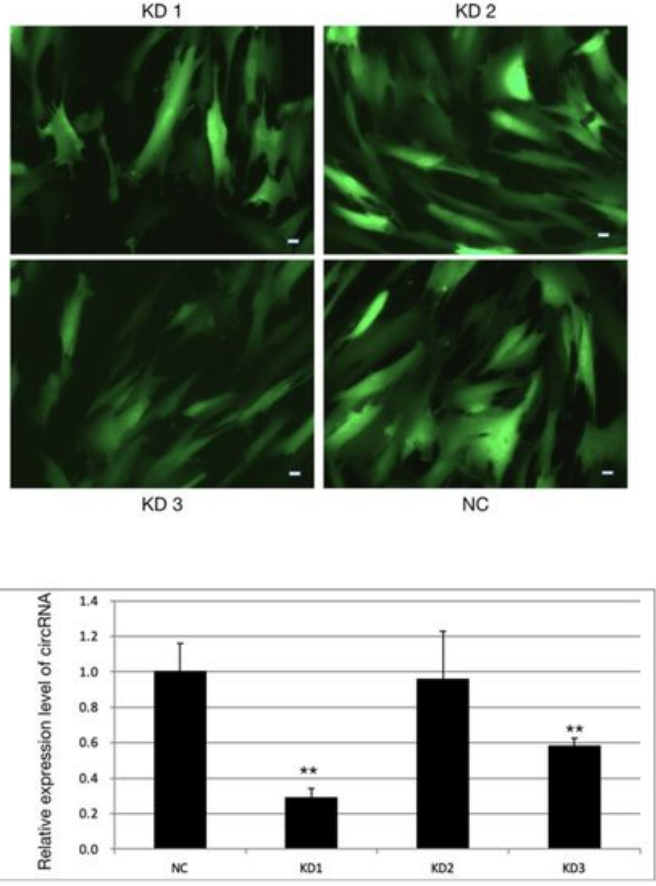

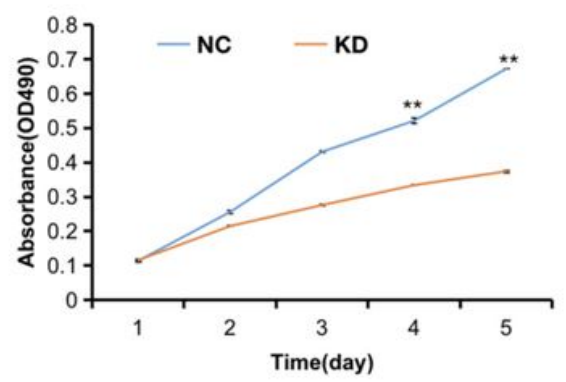

D

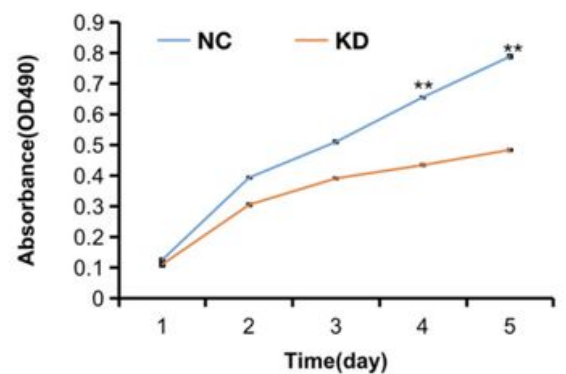

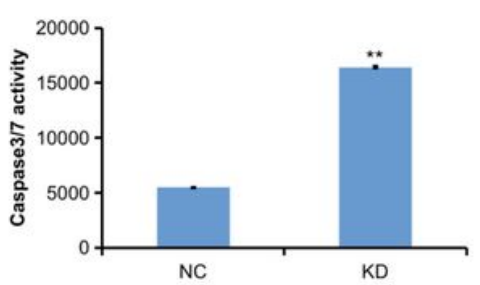
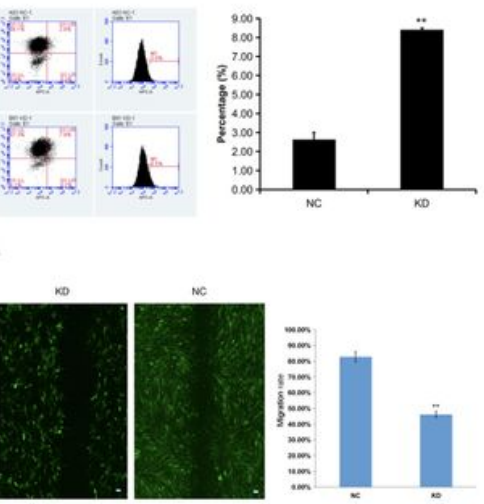

\section{Figure 4}


Knockdown of hsa_circ_0005505 inhibits HBVSMCs proliferation, migration and induces apoptosis. A. HBVSMCs infected by 4 kinds of lentivirus. Scale bar $=50 \mu \mathrm{m}$ B. The infection efficiency of lentivirus assessed by qPCR. C, D. MTT and CCK8 assays of HBVSMCs transfected with hsa_circ_0005505 shRNAs or negative control were performed to evaluate cell proliferation ability. E, F. Caspase 3-7 and flow cytometry apoptosis analysis of HBVSMCs transfected with control or hsa_circ_0005505 shRNAs. G. Wound-healing assay on hsa_circ_0005505 silenced HBVSMCs and control. The graph shows quantification of the residual wounded area $24 \mathrm{~h}$ after scratch. Scale bar $=100 \mu \mathrm{m} . \mathrm{KD}=$ knockdown of hsa_circ_0005505, NC=negative control. * $p<0.05, * \star p<0.01,{ }^{\star \star *} p<$ 0.001.

\section{Supplementary Files}

This is a list of supplementary files associated with this preprint. Click to download.

- Additionalfile1.pdf

- Additionalfile2.pdf

- Additionalfile3.docx

- Additionalfile4.xls

- Additionalfile5.xls

- Additionalfile6.xls 